\title{
O risco elevado do desenvolvimento das doenças crônicas não transmissíveis em cuidadores de pessoas idosas
}

\section{The high risk of the development of chronic non communicable diseases in caregivers of the elderly}

\author{
Vilmar da Conceição Oliveira Filho'• Selma Petra Chaves de Sá • Gabrielle de Souza Rocha \\ Ana Maria Domingos ${ }^{4} \cdot$ Rafael da Silva Soares $^{5} \bullet$ Taianah Almeida Barroso $^{6}$
}

\begin{abstract}
RESUMO
Introdução: A baixa qualidade de vida associada ao sobrepeso e a circunferência abdominal elevada em cuidadores, demonstra ser um fator de risco para o desenvolvimento de doenças crônicas não transmissíveis. Objetivo: Relacionar características sociodemográficas, antropométricas com a qualidade de vida de cuidadores. Método: Estudo transversal, com 3I cuidadores, atendidos no Centro de Atenção à Saúde do Idoso e seus Cuidadores. Responderam um questionário sociodemográfico e realizaram antropometria com as seguintes variáveis: peso, estatura, circunferência abdominal (CAb) e cálculo do índice de massa corporal (IMC), a CAb e o IMC foram classificados segundo a Organização Mundial da Saúde, 2000. A qualidade de vida foi mensurada pela escala SF-36. No tratamento de dados foram realizados os testes de Shapiro Wilk e Mann-Whitney no software SPSS (22.0). Resultados: $45,16 \%$ eram formais, $61,3 \%$ possuíam o ensino médio completo e $38,8 \%$ ganhavam I salário mínimo.A obesidade foi de $35,5 \%$ e de CAb maior que $88 \mathrm{~cm}$ foi 71,1\%. Observou-se que cuidadores, no geral, apresentavam pior qualidade de vida nos domínios dor e estado geral. Conclusão: Os cuidadores apresentaram baixa renda, nível de escolaridade variado, predomínio significativo do sobrepeso e circunferência abdominal elevada.A qualidade de vida apresentou associação com o estado nutricional.
\end{abstract}

Descritores: Cuidadores; Qualidade de Vida; Estado Alimentar; Políticas Públicas

\begin{abstract}
Introduction: The low quality of life associated with overweight and high abdominal circumference in caregivers, demonstrating that it is a risk factor for the development of chronic diseases. Objectives:To relate sociodemographic, anthropometric characteristics to the quality of life of caregivers. Methods: A cross sectional study was carried out with 3I caregivers attended at the Health Care Center of the Elderly and their Caregivers. They answered a sociodemographic questionnaire and anthropometry was performed with the following variables: weight, height, waist circumference $(\mathrm{CAb})$ and body mass index $(\mathrm{BMI})$. CAb and BMI were classified according to the World Health Organization, 2000. Quality of life was measured by the SF-36 scale. Data were analyzed by Shapiro Wilk and MannWhitney in the SPSS software (22.0). Results:That $45.16 \%$ were formal, $61.3 \%$ had completed high school and $38.8 \%$ of them were paid I minimum wages. The frequency of obesity was $35.5 \%$ and the CAb presented $7 \mathrm{I} .1 \%$ over 88 $\mathrm{cm}$. It was observed that the caregivers, in general, presented worse quality of life in the domains pain and general state. Conclusions: Caregivers presented low income, varied educational level, significant predominance of overweight and high abdominal circumference, which indicates cardiovascular risk. Quality of life showed an association of some domains with nutritional status.
\end{abstract}

Keywords: Caregivers; Quality of life; Food State; Public politics. 


\section{INTRODUÇÃO}

Segundo a Organização Mundial de Saúde (OMS), até 2025 a população de idosos crescerá, proporcionalmente, 16 vezes mais do que a população total.' Dados que corroboram com o que encontramos no Brasil no Instituto Brasileiro de Geografia Estatística, em que a população manteve uma tendência de envelhecimento nos últimos anos e ganhou 4,8 milhões de idosos desde 2012, superando a marca de 30,2 milhões em 2017. ${ }^{2}$ Esta realidade acarreta um grande problema social uma vez que essa população, em sua maioria, vive em condições não muito favoráveis.A qualidade a assistência à saúde atrelada ao avançar da idade e aparecimento de enfermidades, têm causado o crescimento de idosos portadores de incapacidades, tornando-se necessária a presença de cuidadores, sejam formais ou informais, para auxiliá-lo em suas limitações. ${ }^{3}$

O cuidador é responsável por zelar pelo cuidado do idoso sadio ou enfermo na realização de suas atividades diárias. ${ }^{4}$ São classificados como formais ou informais, sendo os formais aqueles que irão atuar no mercado de trabalho, já os informais, majoritariamente, são os que desempenham o cuidado de seus respectivos familiares e/ou amigos. ${ }^{5}$ Independentemente de o cuidador ser formal ou informal existe uma tendência predominante da investigação dos efeitos negativos nessa função, com destaque para o estado nutricional, doenças psicossomáticas, ansiedade, depressão e estresse. Portanto, se faz necessário o acompanhamento multidisciplinar almejando garantir a saúde do cuidador. ${ }^{6}$

Sabe-se que o estado nutricional é fundamental na qualidade de vida de todos os seres humanos. Vale ressaltar que a alimentação inadequada prejudica o desempenho, a capacidade funcional e psicológica do cuidador e, consequentemente, os cuidados prestados aos idosos. ${ }^{7}$ A necessidade especial e individual de cada pessoa idosa acaba demandando um cuidado específico de seu cuidador e isso pode interferir na saúde nutricional desta pessoa que assume o cuidado com o idoso. Essa demanda de tempo para desempenhar o cuidado impossibilita uma regularização/orientação de uma alimentação saudável e, principalmente, de qualidade dos alimentos ingeridos. Assim, as atividades realizadas pelos cuidadores principalmente, os informais e/ou familiares, podem interferir no estado nutricional e consequentemente, na sua qualidade de vida. Logo, é imprescindível a atuação do profissional nutricionista através de educação em saúde, orientando uma alimentação saudável afim de promover um peso saudável e bom estado nutricional para a melhoria da qualidade de vida e atividade do cuidador. ${ }^{8}$

Portanto, este estudo teve como objetivo relacionar as características sociodemográficas e antropométricas com a qualidade de vida de cuidadores de pessoas idosas.

\section{MÉTODO}

\section{Delineamento do estudo}

Trata-se de estudo transversal, com amostra aleatória, porém todos os participantes presentes foram do sexo feminino, realizado com 31 cuidadores de idosos no período de fevereiro a junho de 2018, no Centro de Atenção à Saúde do Idoso e Cuidador (CASIC), pertencente à Universidade Federal Fluminense (UFF), localizado em Niterói, Rio de Janeiro. $O$ estudo obedeceu a Resolução $n^{\circ}$. 466/12 do Conselho Nacional de Saúde (CNS), junto ao CAAE, $n^{\circ}$ 76603617.9 .0000 .5243 com número do parecer do Comitê de Ética em Pesquisa da Faculdade de Medicina da Universidade
Federal Fluminense (CEP/FMUFF) $\mathrm{n}^{\circ} 2.386 .997$ e recebeu financiamento da Coordenação de Aperfeiçoamento de Pessoal de Nível (CAPES) pela bolsa de auxílio à pesquisa.

\section{Critérios de Elegibilidade}

Foram incluídas no estudo cuidadores de 20 a 59 anos que já são atendidos no CASIC e excluídas participantes grávidas, aqueles que possuíam marca passo e que já tivessem recebido atendimento nutricional seja no CASIC ou em outro local.

No momento inicial, as participantes responderam um questionário sociodemográfico com as seguintes variáveis: sexo, idade, renda e escolaridade. Posteriormente foi realizada a antropometria com as seguintes variáveis: a circunferência abdominal (CA) em $\mathrm{cm}$, peso e estatura. A circunferência abdominal é definida como sendo a maior medida no nível da cicatriz umbilical no final do movimento expiratório. Os pontos de corte adotados para CA foram os preconizados para a população sul americana onde valores acima de $(C A>80 \mathrm{~cm})$ para mulheres e para homens $(C A>94 \mathrm{~cm})$ estão relacionados com risco de doença cardiovascular. ${ }^{9} \mathrm{~A}$ massa corporal $(\mathrm{kg})$ foi avaliada por meio de uma balança Welmy ${ }^{\circledR}$ com capacidade máxima de $140 \mathrm{~kg}$ e precisão de $100 \mathrm{~g}$ previamente aferida pelo Instituto Nacional de Metrologia (INMETRO); Estatura $(\mathrm{cm})$ que foi aferida com um estadiômetro da marca Welmy ${ }^{\circledR}$ num intervalo operacional de $210 \mathrm{~cm}$ e precisão de $1 \mathrm{~cm}$. Para a verificação da massa e da estatura, as participantes estavam descalças, sem bolsas e objetos no bolso, em pé e com os mesmos juntos sobre a base do estadiômetro.

O Índice de Massa Corporal (IMC) foi calculado com as medidas de peso e altura, de acordo com a seguinte fórmula IMC = peso $(\mathrm{kg}) /$ altura $^{2}(\mathrm{~m})$.A classificação foi feita de acordo com os parâmetros propostos pela Organização Mundial da Saúde, 10 sendo os pontos de corte dispostos da seguinte maneira: desnutrição (IMC $<18,5 \mathrm{~kg} / \mathrm{m}^{2}$ ), eutrofia (IMC $\geq$ 18,5 e $<24,9 \mathrm{~kg} / \mathrm{m}^{2}$ ), sobrepeso (IMC $\geq 25$ e $<29,9 \mathrm{~kg} / \mathrm{m}^{2}$ ), obesidade grau I (IMC $\left.\geq 30 \mathrm{e}<34,9 \mathrm{~kg} / \mathrm{m}^{2}\right)$, obesidade grau II (IMC $\geq 35,0$ e $39,9 \mathrm{~kg} / \mathrm{m}^{2}$ ) e obesidade grau III (IMC $\geq 40 \mathrm{~kg} /$ $\left.\mathrm{m}^{2}\right)$.

Complementando a coleta foi aplicada a escala SF-36, validada, onde se verificam 8 domínios e almeja analisar o status de qualidade de vida. Os dados foram coletados a partir de uma consulta presencial previamente agendada com as cuidadoras por meio de um contato telefônico.

\section{Tratamento dos dados}

As variáveis, índice de massa corporal (IMC) e circunferência abdominal (CA) foram testadas quanto a sua normalidade pelo teste Shapiro Wilk e as que não apresentaram distribuição normal pelo teste de Wilcoxon, uma vez que as. A análise descritiva foi realizada utilizando o cálculo da média, mediana, percentis, desvio padrão e coeficiente de variação. A variabilidade da distribuição de uma variável quantitativa será considerada baixa se CV $<0,20$ moderada se $0,20 \leq \mathrm{CV}<$ 0,40 e alta se $C V \geq 0,40$. Para a comparação da distribuição da variável quantitativa dos grupos independentes foi utilizado o teste não paramétrico de Mann-Whitney. A associação entre duas variáveis quantitativas foi investigada por Análise de Correlação. A intensidade da correlação foi medida pelo Coeficiente de Correlação de Pearson, uma vez que as variáveis não seguiam distribuição normal. Os valores de $\mathrm{p}<$ 0,05 foram considerados significativos. Todas as análises foram feitas utilizando o software Statistical Package for the Social Science (SPSS) versão 22.0. 


\section{RESULTADOS}

Participaram da pesquisa 31 cuidadores, é válido ressaltar que na pesquisa somente aceitaram participar cuidadores do sexo feminino $100 \%(n=31)$, sendo $45,16 \%(n=14)$ classificadas como formais e $54,84 \%(n=17)$ como informais. Em relação às variáveis sociodemográficas, cuidadoras apresentaram faixa etária de 36 a 59 anos $(96,8 \%, n=30), 61,3 \%(n=19)$ possuem o ensino médio completo e $71 \%(n=22)$, recebem 2 salários mínimos ou menos. Esses dados são apresentados na tabela I.

Tabela I: Características sociodemográficas das cuidadoras. Niterói, Rio de Janeiro, Brasil, 2018.

\begin{tabular}{|lcc|}
\hline Variável & Amostra & Total* \\
\hline Escolaridade & & \\
\hline Ensino Fundamental Incompleto & $\mathrm{I}$ & $(3,2)$ \\
\hline Ensino Fundamental Completo & $\mathrm{I}$ & $(3,2)$ \\
\hline Ensino Médio Incompleto & 5 & $(16, \mathrm{I})$ \\
\hline Ensino Médio Completo & 19 & $(61,3)$ \\
\hline Ensino Superior Completo & 5 & $(16, \mathrm{I})$ \\
\hline Renda & & \\
\hline Sem Renda & 4 & $(12,9)$ \\
\hline I salário mínimo & 12 & $(38,8)$ \\
\hline 2 salários mínimos & 6 & $(19,3)$ \\
\hline 3 a 4 salários mínimos & 6 & $(19,3)$ \\
\hline 5 salários mínimos ou mais & 3 & $(9,7)$ \\
\hline
\end{tabular}

*Valores expressos sob forma de número absoluto (percentual).

Quanto aos dados antropométricos, ○ IMC apresentou média e desvio padrão de $29,3 \pm 5, \mathrm{l} \mathrm{kg} / \mathrm{m}^{2}$ e a circunferência abdominal, resultando com $99,0 \pm 8,9 \mathrm{~cm}$ as distribuições de frequências e classificação de risco para doença metabólica segundo a circunferência abdominal apresentam-se na Tabela 2 .

Tabela 2: Características antropométricas das cuidadoras. Niterói, Rio de Janeiro, Brasil, 2018

\begin{tabular}{|lcc|}
\hline Variável & Amostra & Total \\
\hline IMC $\left(\mathrm{kg} / \mathrm{m}^{2}\right)$ & $\mathrm{n}$ & $(\%)^{*}$ \\
\hline $\mathrm{I} 8,5-24,9$ (eutrofia) & 10 & $(32,2)$ \\
\hline $25,0-29,9$ (sobrepeso) & 10 & $(32,2)$ \\
\hline $30,0-34,9$ (obesidade grau I) & 06 & $(19,4)$ \\
\hline $35,0-39,9$ (obesidade grau II) & 04 & $(12,9)$ \\
\hline$\geq 40$ (obesidade grau III) & $0 \mathrm{I}$ & $(3,2)$ \\
\hline Circunferência Abdominal & & \\
\hline$\leq 80 \mathrm{~cm}$ (risco baixo) & 2 & $(6,4)$ \\
\hline $80-87 \mathrm{~cm}$ (risco aumentado) & 7 & $(22,5)$ \\
\hline$>88 \mathrm{~cm}$ (risco muito aumentado & 22 & $(71, \mathrm{I})$ \\
\hline
\end{tabular}

* Número absoluto e proporção ponderada da amostra; IMC: Índice de massa corporal.

A análise da qualidade vida pelos escores apresenta alta variabilidade, mas em termos medianos, observa-se que as cuidadoras têm uma pior qualidade de vida em geral sendo mais baixa nos domínios dor e estada geral. Porém melhor qualidade de vida nos domínios aspectos física, aspectos sociais e saúde mental como visto nos dados apresentados (Tabela 3).
Tabela 3: Comparação entre os grupos obesos e não obesos em relação ao SF-36. Niterói, Rio de Janeiro, Brasil, 2018.

\begin{tabular}{|c|c|c|c|c|}
\hline Domínio & $\begin{array}{l}\text { Total } \\
n=31\end{array}$ & $\begin{array}{c}\text { Não obesos } \\
n=20\end{array}$ & $\begin{array}{c}\text { Obesos } \\
n=I I\end{array}$ & p-valor \\
\hline \multicolumn{5}{|c|}{ Capacidade Funcional } \\
\hline Média & 74,5 & 84,8 & 55,9 & \\
\hline Mediana & 80,0 & 87,5 & 60,0 & $0,001 *$ \\
\hline Desvio Padrão & 22,8 & 14,9 & 23,3 & \\
\hline \multicolumn{5}{|l|}{ Aspectos Físicos } \\
\hline Média & 62,1 & 63,8 & 59,1 & \\
\hline Mediana & 75,0 & 75,0 & 50,0 & 0,855 \\
\hline Desvio Padrão & 32,8 & 29,8 & 39,2 & \\
\hline \multicolumn{5}{|l|}{ Dor } \\
\hline Média & 51,3 & 56,0 & 42,7 & \\
\hline Mediana & 51,0 & $5 I, 5$ & $4 I, 0$ & 0,197 \\
\hline Desvio Padrão & 26,1 & 25,0 & 27,0 & \\
\hline \multicolumn{5}{|l|}{ Estado Geral } \\
\hline Média & 54,0 & 60,4 & 42,5 & \\
\hline Mediana & 57,0 & 64,5 & 47,0 & $0,020 *$ \\
\hline Desvio Padrão & 20,5 & 18,9 & 18,9 & \\
\hline \multicolumn{5}{|l|}{ Vitalidade } \\
\hline Média & 51,1 & 50,5 & 50,5 & \\
\hline Mediana & 45,0 & 47,5 & 45,0 & 0,761 \\
\hline Desvio Padrão & 25,6 & 26,5 & 25,3 & \\
\hline \multicolumn{5}{|l|}{ Aspectos Sociais } \\
\hline Média & 66,5 & 66,9 & 65,9 & \\
\hline Mediana & 75,0 & 75,0 & 50,0 & 0,919 \\
\hline Desvio Padrão & 24,9 & 23,7 & 28,0 & \\
\hline \multicolumn{5}{|c|}{ Aspectos Emocionais } \\
\hline Média & 46,2 & 41,7 & 54,5 & \\
\hline Mediana & 33,3 & 33,3 & 66,7 & 0,476 \\
\hline Desvio Padrão & 41,9 & 38,8 & 47,8 & \\
\hline \multicolumn{5}{|l|}{ Saúde Mental } \\
\hline Média & 61,7 & 59,6 & 65,5 & \\
\hline Mediana & 60,0 & 60,0 & 68,0 & 0,502 \\
\hline Desvio Padrão & 24,7 & 20,1 & 32,2 & \\
\hline
\end{tabular}

*Legenda: p-valor do teste de Mann-Whitney comparando os dois subgrupos.

Os domínios de saúde capacidade funcional, vitalidade e aspectos emocionais apresentaram, resultados próximo aos 50 , que representa a proximidade com a média. $A$ associação entre a qualidade de vida e o estado nutricional, como pode ser observado na Tabela 3, nos domínios de capacidade funcional e estado geral, apresentaram uma diferença significativa entre o grupo de obesos e não obesos.

\section{DISCUSSÃO}

As cuidadoras deste estudo foram, em sua maioria, informais $(54,8 \% ; n=17)$. Independentemente de ser formal ou informal, a atividade realizada pelo cuidador de idoso impacta consideravelmente, na qualidade de vida da pessoa idosa. Uma das funções do cuidador é fornecer suporte para a realização das atividades de vida diária, apoiando o idoso nas suas necessidades físicas, sociais, psicológicas e até econômicas, favorecendo a melhora da qualidade de vida."

$\mathrm{O}$ ato do cuidado ao idoso continua sendo realizado com 
predomínio do sexo feminino, dado esse que corrobora com achados que reforçam o papel social da mulher, em que as atividades que derivam do ato de cuidar, tendem a ser atribuídas a elas e naturalizadas de forma a aparecerem como exclusivas e constitutivas da condição feminina. Este papel pode provocar nesta mulher cuidadora, o descuido de sua própria saúde, nutricional, psicológica e social. ${ }^{12,13,14}$

$\mathrm{Na}$ análise do estado nutricional, constatou-se a predominância dos casos de sobrepeso e obesidade das cuidadoras, semelhante à outra pesquisa na qual foi encontrada prevalência de sobrepeso entre os cuidadores. ${ }^{7}$ Em um estudo $5 \mathrm{I}, 1 \%$ dos cuidadores apresentaram sobrepeso e obesidade. ${ }^{15}$ Estes dados apontam para situação crítica por se tratar de população relativamente jovem e ativa, o que mostra o reflexo dos atuais hábitos alimentares no impacto a saúde. ${ }^{16}$

O excesso de peso/obesidade compromete a saúde dos indivíduos, acarretando em alterações metabólicas, psicológicas, dificuldades respiratórias e de locomoção além de ser fator de risco para enfermidades como: hipertensão arterial sistêmica, diabetes mellitus tipo 2, alguns tipos de câncer, fatores que influenciam na qualidade e no bom desenvolvimento do serviço oferecido por esses indivíduos. ${ }^{17}$

Altos valores de circunferência abdominal foram observados em 93,6\% das cuidadoras aponta para risco elevado do desenvolvimento de doenças cardiovasculares, apresentando resultado superior ao que foi encontrado em outro estudo, que também realizou a mesma aferição demonstrando $67,74 \%$ de desenvolvimento da mesma enfermidade. ${ }^{7}$

Outro estudo mostra que a prevalência de obesidade abdominal tem aumentado nos últimos anos, principalmente em mulheres. Essa maior prevalência em mulheres poderia ser atribuída à maior concentração de gordura corporal comumente relatada devido a gestações, alterações hormonais e ao climatério. Além disso, com o processo de envelhecimento e redistribuição da gordura, as mulheres passam a acumular mais gordura subcutânea. Também se verificou que as mulheres, que possuem valores elevados da circunferência abdominal apresentam um fator de risco independente de morbimortalidade para as doenças crônicas não transmissíveis. ${ }^{16}$

É possível que essa elevada prevalência de excesso de peso e obesidade abdominal encontrada possa ser explicada pela situação social desfavorecida das mulheres estudadas já que a maior parte possuía renda familiar inferior a dois salários mínimos, dado também encontrado em outro estudo em que a maior parte da amostra possuía mesma faixa de renda."

Há evidências de que a qualidade da dieta está intimamente relacionada com a inserção socioeconômica de classe de forma que populações menos favorecidas apresentem ingestão elevada de alimentos de alta densidade, mais baratos e que proporcionam maior saciedade. ${ }^{18}$

Assim, os dados apresentados indicam que os cuidadores, necessitam de orientações multidisciplinares emultiprofissionais que promovam sua saúde atendendo as suas necessidades, atuando na prevenção das doenças e, consequentemente, melhora do seu estado nutricional e qualidade de vida. ${ }^{19}$

$\mathrm{Na}$ avaliação da escala SF-36 de qualidade de vida, à capacidade funcional apresentou o resultado mais significativo no momento em que foi aplicada a escala SF36 onde demonstrou associação com a obesidade, este resultado aponta o encontrado em outro estudo, onde o estado nutricional de obesidade reduziu a capacidade funcional de mulheres adultas. ${ }^{20}$

No domínio vitalidade, as cuidadoras apresentaram resultado médio de 54,4 pontos, o que caracteriza um resultado médio na capacidade de execução das tarefas do cuidado e atividades diárias. $O$ resultado citado corrobora com o encontrado por outro estudo, em pesquisa realizada no estado de Pernambuco onde a média do domínio apresentou 48,8 pontos de resultado e predomínio do cuidado realizado pelo sexo feminino com $86 \%$ da amostra. ${ }^{21}$

As cuidadoras apresentaram um resultado geral relativamente baixo no cálculo do domínio dor, em que obtiveram média de 52,5, o que caracteriza sofrimento na execução das atividades diárias, corroborando com o que foi encontrado na literatura científica. ${ }^{22}$ Tal fato aponta para a necessidade do cuidado do profissional com a saúde do cuidador, conhecer o seu perfil e as condições às quais estão expostos, pois o mesmo pode desenvolver alguma enfermidade como lesões na coluna cervical ou dorsal devido à sobrecarga da rotina como demonstram o estudo realizado no Centro de Referência Estadual de Atenção à Saúde do Idoso (CREASI), Salvador. ${ }^{23}$

O estado geral da saúde apresentou resultado não favorável. A obesidade pode ter efeitos negativos em longo prazo, na qualidade de vida, pois o aumento do peso interfere no estado geral da saúde. ${ }^{21} \mathrm{O}$ estado nutricional de sobrepeso e obesidade vem ocupando lugar de destaque na agenda pública internacional e a Organização Mundial da Saúde (OMS) consideram a obesidade como uma epidemia mundial.

No Brasil, o sobrepeso e a obesidade vêm aumentando em todas as faixas etárias e em ambos os sexos, em todos os níveis de renda, sendo a velocidade de crescimento mais expressiva na população com menor rendimento familiar. ${ }^{22}$ O estado nutricional do sobrepeso e obesidade eleva o risco do surgimento das doenças crônicas não transmissíveis (DCNT) afetando a qualidade de vida do indivíduo, fato que foi observado em uma pesquisa que o estado nutricional de sobrepeso e obesidade apresentou uma redução no domínio da capacidade funcional.,

No domínio aspecto emocional da escala de qualidade de vida, que avaliou o quanto a saúde do cuidador encontravase comprometida, o resultado resultou em, 54,2 pontos, fatos observados, muita das vezes, devido ao isolamento social pela rotina enfrentada. Do mesmo modo, no domínio saúde mental, a média foi de 6I,7 que indicou a sobrecarga psicológica que as cuidadoras estavam sofrendo.

O resultado em ambos os domínios indica sobrecarga no ato do cuidado, estresse e isolamento social. A sobrecarga elevada também foi encontrada em outras pesquisas realizadas onde o baixo nível de renda indicou alta sobrecarga das cuidadoras do grupo com média de idade. 13,21,22,24

Os domínios que apresentaram resultados relativamente considerados dentro de referência, foram o de aspectos sociais, o que representa o nível de bem-estar social do cuidador em relação a frequência de contatos e relação com amigos ou familiares. $O$ mesmo ocorreu nos dados referentes aos aspectos físicos com pontuação de 63,5, o qual permite verificar a qualidade da atividade física ou motricidade do sujeito. Os respectivos resultados corroboram com pesquisa realizada na cidade de $\mathrm{Natal} / \mathrm{RN}$, a qual avaliou a qualidade de vida dos cuidadores. ${ }^{25}$

O cuidado com o cuidador deve ter um cunho multidisciplinar objetivando garantir à saúde como todo. Isto 
se justifica, pois, os cuidadores estão sujeitos às enfermidades psicológicas e físicas como a depressão, sobrepeso, obesidade, ansiedade, isolamento social, inatividade física e inadequação alimentar. Pesquisa realizada no Rio de Janeiro identificou a elevada taxa das doenças crônicas não transmissíveis em cuidadores. Outro estudo identificou em São Paulo a necessidade do cuidado multidisciplinar como o da psicologia, já outra pesquisa realizada em 2018, identificou limitações psicológicas e físicas em cuidadores no Rio Grande do Sul. ${ }^{26,27}$

\section{CONCLUSÃO}

A partir desse estudo evidenciou-se que o perfil sociodemográfico de cuidadores de pessoas idosas é formado majoritariamente, por cuidadores informais e do sexo feminino. Ao ser avaliado o estado nutricional, constatou-se a predominância dos casos de sobrepeso e obesidade nas cuidadoras e altos valores de circunferência abdominal. Tais índices enfatizam o risco do desenvolvimento de alterações metabólicas e sistêmicas ao longo da vida, elevando o risco para o desenvolvimento de doenças crônicas não transmissíveis.

A aplicação de escala especializada para avaliação da qualidade de vida permitiu evidenciar redução da capacidade funcional e sua associação com a obesidade, sofrimento na execução das atividades diárias e sua relação com a rotina de trabalho diária desenvolvida no cuidado com a pessoa idosa, além de indicadores que confirmam estresse e isolamento social, culminando com estado geral da saúde não favorável, principalmente nos obesos.

Esse panorama sugere que os cuidadores de pessoas idosas se mostram em situação de vulnerabilidade, estando sujeitos a enfermidades físicas, biológicas e psicológicas. Portanto, fazemse necessárias ações de saúde e apoio social à cuidadores de pessoas idosas, garantindo e promovendo a implantação de políticas públicas e sociais direcionadas para esses indivíduos. As ações de saúde devem primar por uma abordagem multidisciplinar e multiprofissional, cujas ações promovam saúde e estejam de acordo com as suas necessidades específicas, melhorando não apenas o estado nutricional, mas também a qualidade de vida e minimizando o risco do desenvolvimento de doenças crônicas não transmissíveis.

\section{REFERÊNCIAS}

1. Organização Mundial da Saúde. Global health risks: mortality and burden of disease attributable to selected major risks. Genebra: OMS; 2009.

2. Instituto Brasileiro de Geografia e Estatística. Estatísticas sociais. [citado em 18 agosto 2019]. Disponível em: http:www.ibge.gov.br.

3. Araujo JS, Vidal GM, Brito FN, Gonçalves DCA, Leite DKM, Dutra CDT et al. Perfil dos cuidadores e as dificuldades enfrentadas no cuidado ao idoso, em Ananindeua, PA. Rev bras geriatr gerontol. 2013; 16(I): 149-58.

4. Ministério da Saúde. Portaria $n^{\circ} 1395$, de 10 de dezembro de 1999. Aprova a Política Nacional de Saúde da Pessoa Idosa. [citado em 19 agosto 2019]. Disponível em: http://dtr2004.saude.gov.br/susdeaz/ legislacao/arquivo/Portaria_1395_de_10_12_1999. pdf.

5. Silva ILC, Lima GS, Storti LB, Aniceto P, Formighieri PF, Marques S. Sintomas neuropsiquiátricos de idosos com demência: repercussões para o cuidador familiar. Texto contexto enferm 2018; 27(3).

6. Gaioli CCLO, Furegato ARF, Santos JLF. Perfil de cuidadores de idosos com doença de Alzheimer associado à resiliência. Texto contexto enferm 2012; 2 I (I): I50-7.

7. MacielAP, Luna PF,AlmeidaTTG, Carvalho EP.Qualidade de vida e estado nutricional de cuidadores de idosos dependentes. Rev Kairós. 20I5; 18(4): I79-96.

8. Aquino ACF, Alves BMS, Pinho L. Percepção dos usuários sobre as ações educacionais de alimentação e nutrição no Sistema Único de Saúde. Rev baiana saúde pública. 2016; 40(4): 991-1003.

9. Alberti KG, Eckel RH, Grundy SM, Zimmet PZ, Cleeman JI, Donato KA et al. Harmonizing the metabolic syndrome: a joint interim statement of the International Diabetes Federation Task Force on Epidemiology and Prevention; National Heart, Lung, and Blood Institute; American Heart Association; World Heart Federation; International Atherosclerosis Society; and International Association for the Study of Obesity. Circulation. 2009; I 20( I6): 1640-5

10. Organização Mundial da Saúde. Physical Satatus: The Use and Interpretation of Antropometry. Genebra: OMS; 1997.

11. Jesus ITM, Orlandi AAS, Zazzetta MS. Sobrecarga, perfil e cuidado: cuidadores de idosos em vulnerabilidade social. Rev bras geriatr gerontol 2018; 2 I (2): 194-204.

12. Anjos KF, Boery RNSO, Pereira R. Qualidade de vida de cuidadores familiares de idosos dependentes no domicílio. Texto contexto enferm. 20I4; 23(3): 600-8.

13. Leite BS, Camacho ACLF, Jacoud MVL, Santos MSAB, Assis CRCA, Joaquim FL. Relação do perfil epidemiológico dos cuidadores de idosos com demência e a sobrecarga do cuidado. Cogitare enferm. 2017; 22(4).

14. Ferreira CR, Isaac L, Ximenes VS. Cuidar de idosos: um assunto de mulher? Est inter psicol. 2018; 9(I): 108-25.

15. Murta NMG, Miranda LS, Nunes NLA, Almeida TL. Perfil socioeconômico, nutricional e de saúde dos participantes do curso de cuidadores de idosos do Vale do Jequitinhonha/MG. Rev Kairós 2008; I I (2): I 2 I-39.

16. Barroso TA, Marins LB, Alves R, Gonçalves ACS, Barroso SG, Rocha GS. Associação entre a obesidade central e a incidência de doenças e fatores de risco cardiovascular. Int j cardiovasc sci. 20 I 7; 30(5): 4I6-24.

17. Souza LR, Hanus JS, Libera LBD, Silva VM, Mangili EM, Simões PW et al. Sobrecarga no cuidado, estresse e impacto na qualidade de vida de cuidadores domiciliares assistidos na atenção básica. Cad saúde colet. 20I5; 23(2): I40-9.

18. Cristóvão MF, Sato APS, Fujimori E. Excesso de peso e obesidade abdominal em mulheres atendidas em unidade da estratégia saúde da família. Rev esc enferm USP. 20 I I; 45(2): I667-72.

19. Brum AKR, Camacho ACLF, Valente GSC, Sá SPC, Lindolpho MC, Louredo DS. Programa para cuidadores de idosos com demência: relato de experiência. Rev bras enferm. 2013; 66(4): 619-24.

20. Gesser AF, Demartino AM, Oliveira DF, Borges Junior NG, Domenech SC, Gevaerd MS. Qualidade de vida em mulheres com peso normal, sobrepeso e obesidade: uma perspectiva subjetiva e individual. Rev baiana 
saúde pública. 20I4; 38(4): 897-912.

21. Cesario VAC, Leal MCC, Marques APC, Claudino KA. Estresse e qualidade de vida do cuidador familiar de idoso portador da doença de Alzheimer. Saúde debate. 2017; 4I(II2): 17I-82.

22. Fernandes BCW, Ferreira KCP, Marodin MF, Val MON, Fréz AR. Influência das orientações fisioterapêuticas na qualidade de vida e na sobrecarga de cuidadores. Fisioter mov. 2013; 26(I): I5I-8.

23. Vaz LCS, Santos KOB, Ferraz DD. Condições de saúde e trabalho entre cuidadores de idosos frágeis. Rev pesqui fisioter 2018; 8(3): 319-29.

24. Muniz EA, Freitas CASL, Oliveira EN, Lacerda MR. Grau de sobrecarga dos cuidadores de idosos atendidos em domicílio pela estratégia saúde da família. Saúde debate. 2016; 40(110): 172-82.

25. Barbosa LM, Noronha K, Spyrides MHS, Araujo CAD. Qualidade de vida relacionada à saúde dos cuidadores formais de idosos institucionalizados em Natal, Rio Grande do Norte. Rev bras estud popul. 2017; 34(2):
$39|-4| 4$.

26. Silva MIS, Alves ANO, Salgueiro CDBL, Barbosa VF. Doença de Alzheimer: repercussões biopsicossociais na vida do cuidador familiar. Rev enferm UFPE on line. 2018; 12(7): 1931-9.

27. Steindorff GM, Oliveira Junior SB, Viana DR, Maidana Junior JN, Tier CG, Silva VAM. Sobrecarga dos cuidadores de idosos: relato de experiência. Sanare 2018; $17(1):|25-3|$.
Recebido: 2019- 09-18

Aceito: $2019-11-25$ 